\title{
Complutum
}

ISSN: 1131-6993

\section{Cerámica, prácticas técnicas y estructura social mapuche: un caso de dinamismo cultural}

\author{
Jaume García Rosselló ${ }^{1}$
}

Recibido: 1 de julio de 2016 / Aceptado: 1 de marzo de 2017

Resumen. La práctica alfarera mapuche se caracteriza por su alto dinamismo, un fenómeno que ha caracterizado los sistemas de fabricación en la zona desde el siglo XIX. Siguiendo las propuestas de Pierre Lemonnier sobre la representación tecnológica de las estructuras que interactúan en los sistemas técnicos, se analiza el dinamismo documentado en las prácticas técnicas alfareras en tres niveles: la escala interna, como transversalidad tecnológica y en su vinculación con las dinámicas sociales. Como conclusión se plantea que este dinamismo está reflejado de múltiples formas y en diferentes ámbitos, siendo una característica intrínseca del propio grupo que, por ello, afecta a todas las esferas de la sociedad.

Palabras clave: Prácticas técnicas; cerámica; mapuche; dinamismo social.

\section{[en] Pottery, Technical Practices and Mapuche Social Structure: a Case of Cultural Dynamism}

Abstract. Mapuche pottery practice is defined by its high dynamism, typical of these manufacturing systems since the $19^{\text {th }}$ century. The model proposed by Pierre Lemonnier for the technologicalrepresentation of the structures interacting in technical systems can be useful to analyse the dynamism documented in pottery technical practices. Thus, he describes three levels: the internal scale, as a technological transversal entity, and connected to social dynamics. In this case, it may be concluded that this dynamism is materialised in multiple forms and in different areas, constituting a defining element of the group which affects the complete social world.

Keywords: Technical Practices; Pottery; Mapuche; Social dynamism.

Sumario. 1.Planteamiento inicial. 2. La tecnología como construcción social. 3. Estrategia de estudio. 4. Contexto de producción. 5. Estrategias técnicas en la fabricación cerámica. 6. Dinámicas a escala interna: interacción entre las operaciones técnicas. 7. Interacción de los procesos tecnológicos: la transversalidad tecnológica. 8. Interacción entre dinámicas técnicas y acciones sociales: flexibilidad social y organización territorial. 8.1. Organización territorial y social. 8.2. Autonomía y aprendizaje. 9. Conclusiones.

Cómo citar: García Rosselló, J. (2017): Cerámica, prácticas técnicas y estructura social mapuche: un caso de dinamismo cultural. Complutum, 28(2): 341-357.

\section{Planteamiento inicial}

Sin entrar en discusiones sumamente teóricas, pensamos que la mejor manera de definir aquella práctica científica que entendemos como etnoarqueológica es desde el trabajo de campo y del enfoque adoptado en la lectura de los resultados obtenidos. Más allá de definiciones estériles que solo pueden llevar a la fragmentación -aún más, si cabe- de las ciencias sociales, creemos que la manera en la que nos posicionamos en nuestros trabajos podría definirse como etnoarqueología, pero también como arqueología, etnografía, sociología, et-

\footnotetext{
Grupo de investigación ArqueoUIB. Universidad de las Islas Baleares (España)
}

E-mail: jaume.garcia@uib.es 
nohistoria.... En todo caso, la definición que más se ajustaría a la perspectiva que queremos tratar aquí sería la arqueología del presente (González-Ruibal 2016) y los estudios sobre cultura material (Thomas 2004).

Por otra parte, consideramos que si hay un elemento definitorio en nuestros trabajos es la huida recurrente de las analogías de tipo social hechas hacia el pasado y, en general, a otros grupos del presente. Ello no significa que renunciemos a cualquier tipo de analogía, parte fundamental del análisis arqueológico (Gándara 2006), sino que el análisis del contexto es esencial para entender las dinámicas de cualquier grupo social $\mathrm{y}$, por tanto, las mismas no son directamente extrapolables a otras situaciones (Hodder 1982).

En este sentido, se utiliza una perspectiva centrada en el estudio de la materialidad, empleando una metodología fundamentalmente arqueológica bajo un enfoque etnográfico. Todo ello, con el simple objetivo de aportar nuevos datos que ayuden a comprender mejor la sociedad mapuche, en nuestro caso, y contribuir a la discusión de las relaciones existentes entre la materialidad y las dinámicas sociales, teniendo presente la constante interacción entre personas y cosas, y su imposible separación en dos esferas individuales (Olsen 2010, Hernando y González-Ruibal 2011).

Para todo ello, es necesario no abandonar el punto central de cualquier estudio social: el contexto, tal como se ha consensuado en arqueología en la últimas décadas (Hodder 1982). Poner el foco en el análisis del contexto significa tener en cuentas las dinámicas históricas y territoriales. Implica también huir de la viñeta etnográfica -tan común en los trabajos etnoarqueológicos- evitando mostrar una foto fija aislada en tiempo y espacio, que no permite profundizar en las dinámicas que intervienen en una situación social concreta. Si los grupos sociales están en constante construcción y aceptamos que la identidad y la manera de estar en el mundo son contingentes, es necesario incorporar la perspectiva diacrónica en nuestros trabajos sobre los grupos del presente.

\section{La tecnología como construcción social}

Este trabajo aborda la relación existente entre las actuaciones técnicas y las conductas sociales, en tanto que el proceso tecnológico es una construcción social (Calvo y García Rosselló
2014). El comportamiento tecnológico de las personas es esencialmente colectivo, en la medida que está constituido por la totalidad de las operaciones conocidas y estructuradas socialmente (Leroi-Gourham 1964). Las personas aprenden y reproducen las técnicas desde la infancia, y a través de ellas fabrican los objetos. Junto al aprendizaje de una "manera de hacer" se incorporan otros conocimientos que estructuran la "manera de ser" dentro del grupo social y adquieren sentido dentro de él. De esta forma, las técnicas son un vehículo para conectar los objetos, las personas y los valores sociales. Si las técnicas están estructuradas y aceptadas socialmente, la práctica de las mismas, al formar parte de la cotidianeidad, también condiciona y estructura la mentalidad y la manera de estar en el mundo de las personas, de tal modo que son incorporadas a los valores del grupo dentro del que participan.

Es por ello que la lógica tecnológica tendrá sentido únicamente dentro de la lógica social del grupo. En este sentido, planteamos que la tecnología cerámica mapuche es fruto de un conjunto de relaciones sociales negociadas a lo largo del tiempo con las que está fuertemente integrada de forma indisoluble y que, a su vez, estructuran dicha práctica. Se trata de integrar una "lógica tecnológica interna" (el saber hacer, las elecciones y gestos técnicos) con una "lógica tecnológica contextual" (aprendizaje, familia, relaciones sociales, cosmovisión,...) (Mahias 1993). Si bien, esta separación tiene mucho de artificial y de estrategia de investigación, ya que en el mundo real la mutua relación entre ambas "lógicas" es continua, dinámica, estructurada y estructurante (Calvo y García Rosselló 2014).

En el caso de la cerámica mapuche, existe una práctica en la que coexisten actuaciones técnicas estandarizadas con otras que varían aleatoriamente entre las alfareras y que, pese a ello, deben considerarse como parte significativa de la tradición técnica (García Rosselló y de Carvalho 2015). De este modo, estamos convencidos que no existen procesos, acciones y gestos técnicos que puedan ser generalizables entre diferentes sociedades, ni del presente, ni del pasado. Como veremos, a diferencia de otros contextos, el dinamismo existente en la práctica alfarera es característico de una manera de hacer trasmitida durante generaciones y forma parte de la tradición tecnológica del grupo (García Rosselló 2008, 2009). Esta situación no es diferente de las prácticas desa- 


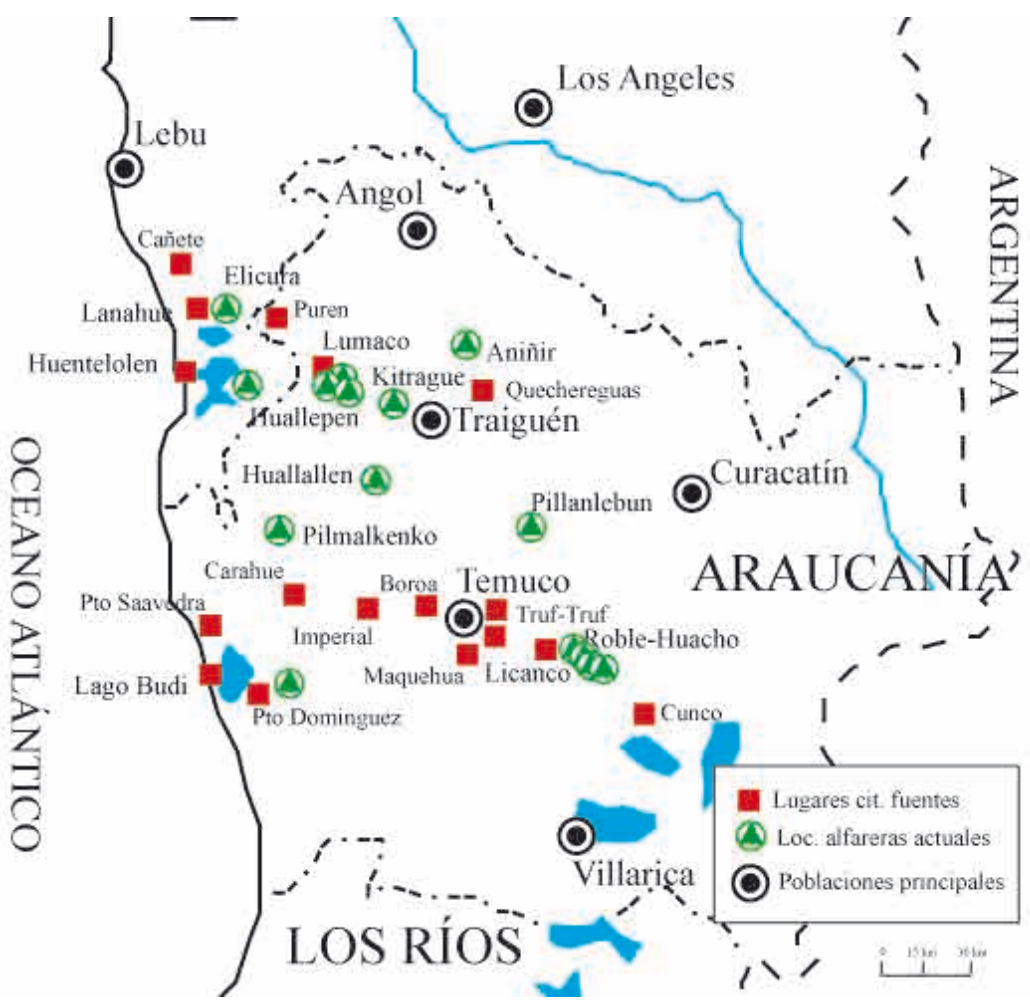

Figura 1. Mapa de localización de las alfareras y comunas prospectadas.

rrolladas en otros campos de la sociedad y en otras tecnologías, convirtiéndose en una característica no solo de la actividad cerámica sino en algo intrínseco al propio grupo que, por ello, afecta a múltiples esferas de la sociedad.

\section{Estrategia de estudio}

Siguiendo con este planteamiento, vamos a articular una discusión fundamentada en el análisis de las técnicas cerámicas de los grupos mapuche, integrándolas dentro de los comportamientos de otras prácticas técnicas como la construcción de la vivienda (ruka) o la confección textil.

Todas estas prácticas técnicas se desarrollan en el día a día, en el seno de contextos domésticos, reproduciendo (y variando) esquemas aprendidos dentro de la tradición, y que están fuertemente vinculados con otros comportamientos sociales.

El uso del concepto de cadena operativa lo hemos enfocado hacia el análisis del proceso técnico y de cada una de las acciones que lo conforman (García Rosselló y Calvo 2012, García Rosselló 2007, 2008, 2011). No se trata simplemente de utilizar una estrategia donde las diferentes etapas del proceso constructivo aparezcan organizadas de forma temporal para facilitar el discurso. Lo que permite este tipo de aproximaciones es analizar el átomo mínimo de acción (el gesto) e integrarlo dentro de actuaciones más amplias, donde la suma de gestos se convierte en técnicas, secuencias de acciones, y uso y selección de materiales en un espacio/tiempo determinado. Así, desglosamos los movimientos, posturas y gestos asociados a personas y a productos en una cadena de acciones que se puede comparar a múltiples niveles acercando o alejando la lente con la que consideramos los diferentes análisis de los comportamientos técnicos.

Así, el análisis de microescala permite relacionar la tecnología con las transformaciones sociales de macroescala. Dicha estrategia, junto a la aproximación contextual, posibilita una mejor interpretación de las relaciones entre las dinámicas sociales y las personas (Calvo y García Rosselló 2014). De hecho, para estudiar la dimensión social del fenómeno tecnológico, no es posible ignorar las acciones técnicas, físicas y reales que se ejecutan sobre la materia. De igual modo, no podemos considerar que el proceso tecnológico sea meramente la secuenciación de acciones físicas, ya 


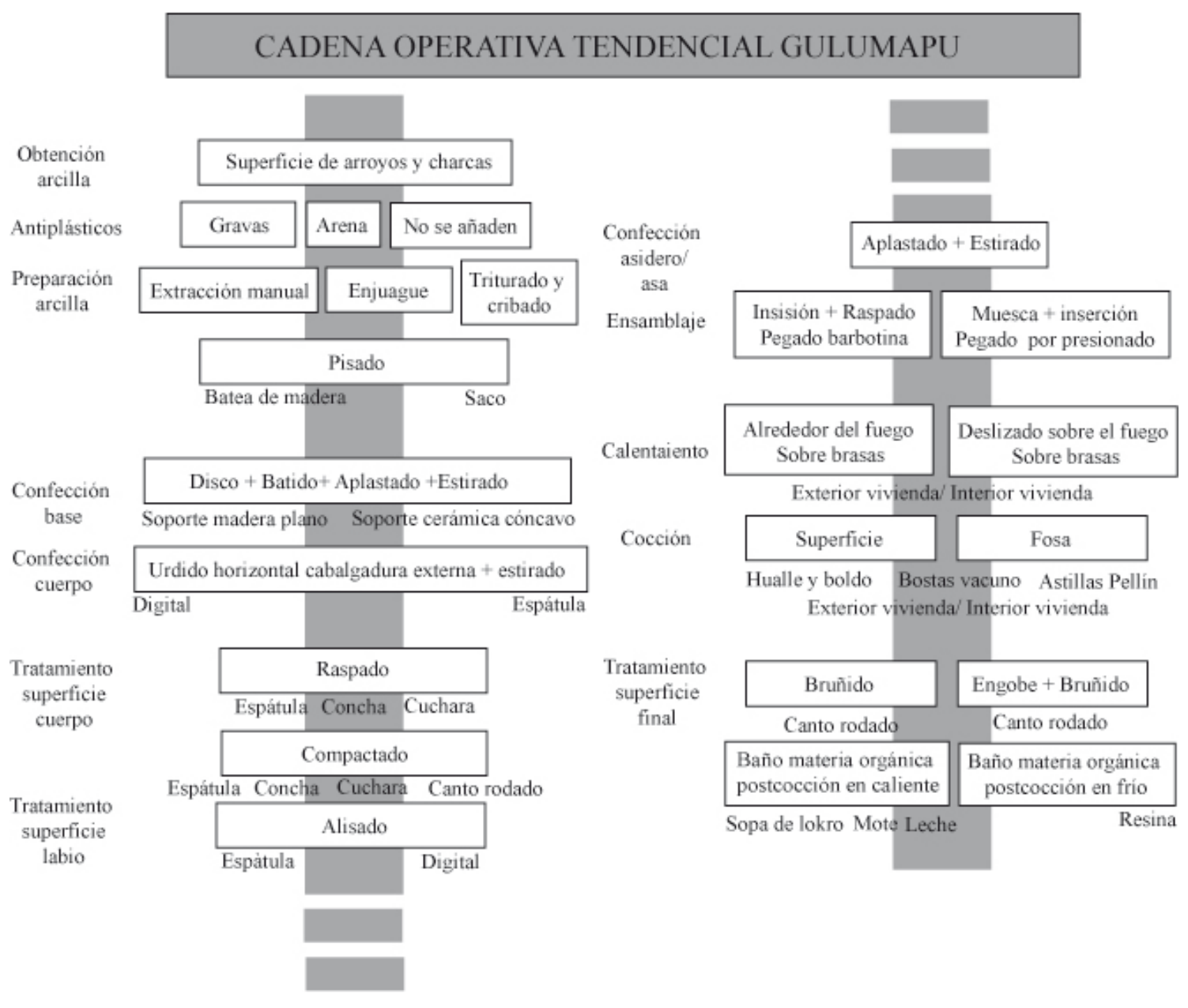

Figura 2. Cadena operativa tendencial de la cerámica mapuche.

que, como hemos dicho, incorpora aquellos esquemas mentales aprendidos a través de la tradición (Lemonnier 1992, 1993).

Para ello utilizamos dos conceptos procedentes de la obra de Lemonnier (1992): el de systèmes technologiques y el de representation (Calvo y García Rosselló 2014). El sistema tecnológico interactúa, a su vez, a tres niveles: 1) la interacción de las dinámicas técnicas a escala interna, dentro de la propia lógica de la secuencia de acciones; 2) la interacción de las dinámicas técnicas con el resto de procesos tecnológicos, es decir, la transversalidad tecnológica; y 3) la interacción de las dinámicas técnicas -tanto a nivel interno como externocon otras dinámicas sociales. De esta forma, profundizamos en los mecanismos mentales que actúan en las personas para tomar decisiones sobre las acciones técnicas, que se encuentran dentro de una red de significados incluida en los esquemas ideológicos, sociales o simbólicos de una comunidad y que han sido definidos como representation technologique por Pierre Lemonnier.

Los datos con los que sustentamos esta discusión han sido recogidos en diferentes campañas de campo entre los años 2007 y $2015^{2}$. Hasta el momento, se han identificado 21 alfareras, ubicadas en las provincias de Arauco, Malleco y Cautín (Figs. 1 y 2$)^{3}$, que mantienen una práctica tradicional trasmitida dentro del núcleo familiar. En total, hemos realizado más de 35 entrevistas que se han desarrollado adaptando la metodología antropológica de historias de vida y del método genealógico. Igualmente hemos seguido algunas de las propuestas definidas anteriormente por nosotros: estrategia productiva y observación participante, en el caso de la documentación de las cadenas operativas. 


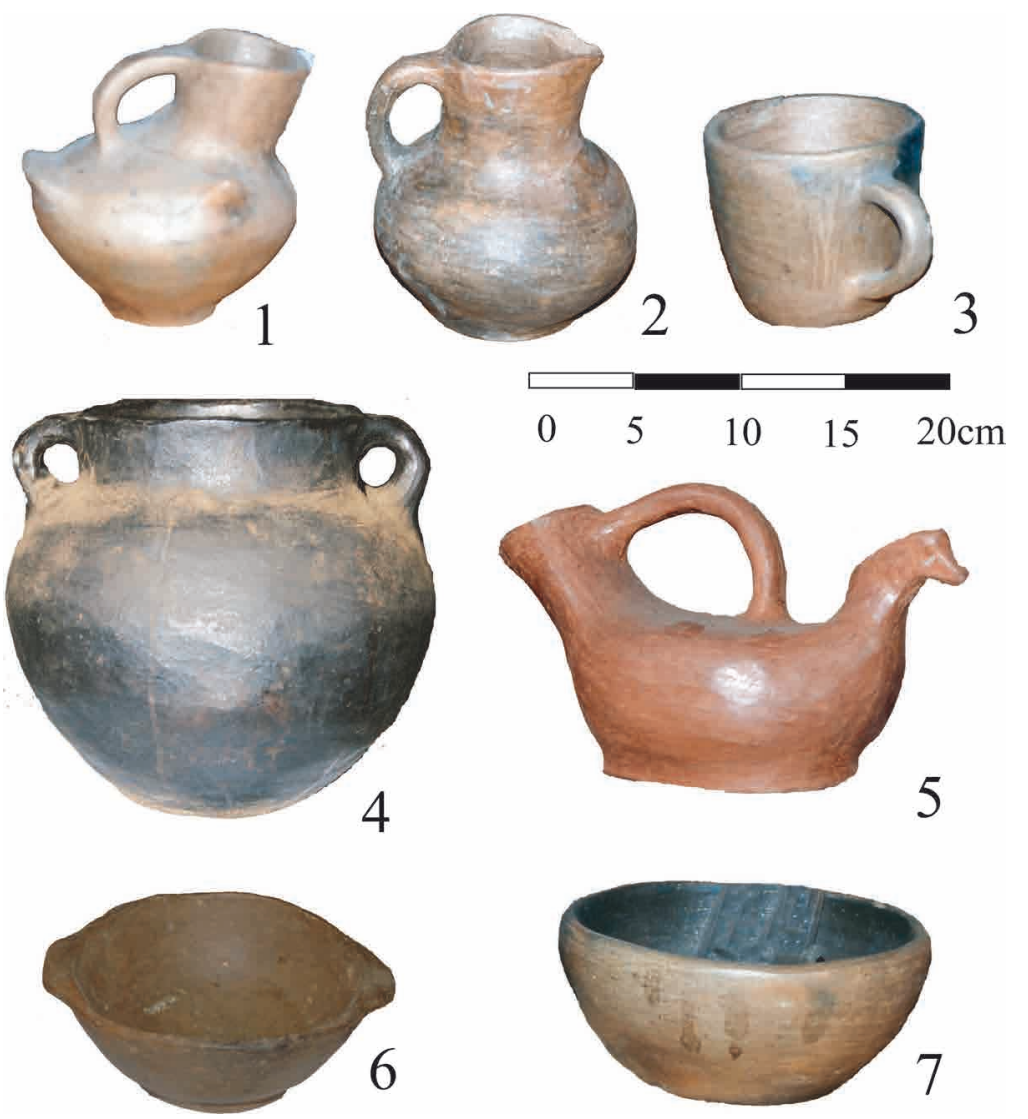

Figura 3. Formas cerámicas: 1.- Ketro metawe. 2.- Pichi metawe. 3.- Taza. 4.- Challa. 5.- Ketro metawe. 6.- Paila. 7.- Rali.

\section{Contexto de producción}

La alfarería mapuche es una actividad fundamentalmente femenina, que se desarrolla y aprende dentro de redes de trasmisión familiares en el seno de la casa y en alternancia con otras actividades domésticas. La venta de estos productos no genera un elevado nivel de ingresos, su práctica se circunscribe al periodo estival y se combina con otras tareas adscritas a las mujeres tales como la crianza, el cuidado de pequeños huertos y el trabajo en la cocina (García Rosselló 2008, de Carvalho y García Rosselló 2013, García Rosselló y Carvalho 2015). Además, convive con la realización de otras artesanías, como el trenzado de voqui ${ }^{4}$, el telar, el trabajo de la madera, o la confección de platería, también presentes dentro del grupo familiar.

En los últimos 20 años la alfarería se ha ido trasformando en una actividad marginal, practicada por un número muy reducido de mujeres, con una media de edad que, en la actualidad, supera los 50-60 años y sin visos de continuidad al no tener aprendices a su cargo de forma permanente.

Estas alfareras están normalmente ubicadas en reducciones ${ }^{5}$ de difícil acceso y se mueven en un ámbito local muy limitado (Course 2011), lo que dificulta el contacto entre ellas, con la excepción de la comunidad de Roble Huacho, en los alrededores de Temuco.

Se trata de una práctica que, pese a las trasformaciones sociales, territoriales y materiales que han protagonizado los grupos mapuche en los últimos 500 años, hunde sus raíces en tiempos prehispánicos (García Rosselló 2009). Mientras las técnicas han permanecido más estables, algunas formas fabricadas y sus decoraciones se han ido trasformando con los años (Alvarado 1997, García Rosselló 2008, García Rosselló y de Carvalho 2015, Adan et al. 2016), a la vez que se han ido mantenido otras, mientras desaparece la fabricación de grandes 

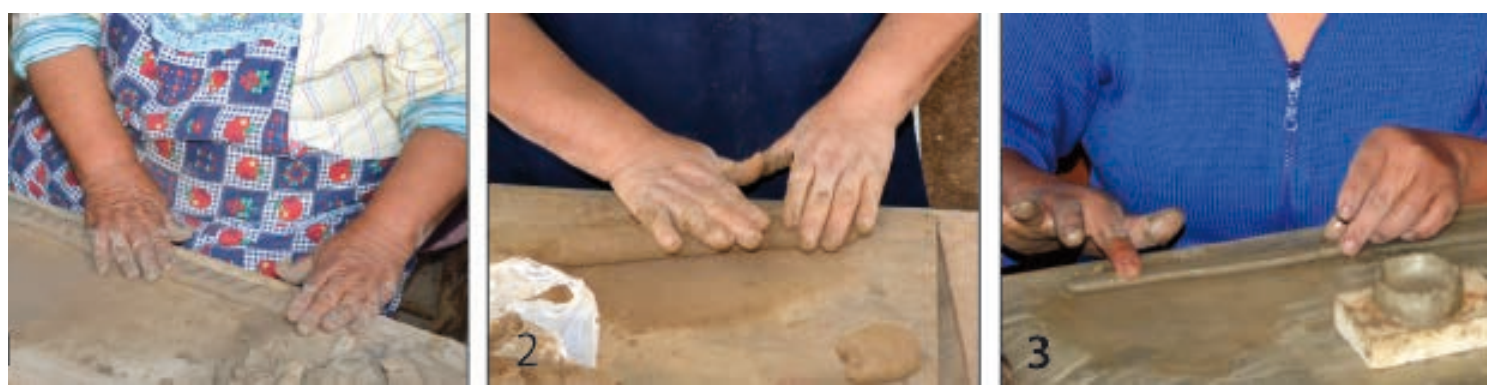

Figura 4. Sistemas de urdido y aplanado de los colombinos: helvetia Cayupán, Maria Cachaña y Ema Tranamil.

contenedores y aquellas vasijas utilizadas para la cocción y almacenaje de alimentos (Alvarado 1997, Adan et al. 2016) (Fig. 3).

Algunas formas ancestrales, como el piche metawe, el metawe o el ketro metawe y otros motivos zooorfos se siguen utilizando (Fig. 3), dada su fuerte carga simbólica, en ceremonias como el we tripantu ${ }^{6}$, el machitún ${ }^{7}$ o el ngillatún $^{8}$ (Course 2011, Dillehay 2011). Es precisamente la demanda de productos por parte de las diferentes comunidades vecinas para sus ceremonias -y no para usos culinarios o de almacenaje-, la que genera un mercado en el que las alfareras distribuyen este tipo de cerámicas mediante pedidos directos. En otros casos, las alfareras ofrecen sus productos, si bien de forma muy esporádica, en ferias y festividades locales, en tiendas de museos y en unos pocos mercados. En este caso, el contacto con población wink $a^{9}$ ha generado la aparición de nuevos tipos, fruto de las demandas existentes y de las dinámicas de mercado: chanchos, platos, tazas, macetas, pailas $^{10}$ que, no obstante, mantienen una raíz fundamentalmente indígena (Fig. 3). Este fenómeno es conocido desde los primeros siglos de ocupación hispánica (Campbell 2012, Adán 2014, Saurer 2015).

\section{Estrategias técnicas en la fabricación cerámica}

La tradición cerámica mapuche se caracteriza por una práctica técnica en la que coexisten ciertas actuaciones homogéneas entre las alfareras, con otras que varían aleatoriamente (Fig. 2). A diferencia de otros contextos, la variabilidad técnica es una característica intrínseca de esta práctica, trasmitida durante generaciones $\mathrm{y}$ que podemos rastrear al menos desde finales del siglo XIX. Se trata de una práctica asociada a los grupos indígenas que antes y después de la llegada de los españoles poblaban el este territorio, como se ha demostrado al comparar los datos históricos de las actuaciones técnicas con las prácticas de las alfareras actuales (García Rosselló 2009). Existen referencias de algunos comportamientos técnicos desde el siglo XVIII y, principalmente, desde 1850 realizadas por eruditos locales, misioneros y viajeros (García Rosselló 2008, García Rosselló y de Carvallo 2015).

$\mathrm{Su}$ extensión abarca todo el Gulumapu, el territorio de residencia de los distintos grupos mapuche situados al oeste de los Andes y que se sitúa entre el golfo de Reloncaví y el río Maipo (Marimán et al. 2006, León 2008). En la actualidad, los grupos rurales que se autoidentifican como mapuches ocupan las provincias de Arauco, Malleco, Cautín y Valdivia, en las regiones VIII, IX y XIV, extendiéndose algo más al sur en algunos casos (Bengoa 1991, 2003).

En general, las alfareras (widüfe en mapundungun) utilizan la misma secuencia encadenada de actuaciones técnicas (Figs. 2, 4 y 5). Esta secuencia se inicia con la recogida de la greda o arcilla y finaliza con algunos tratamientos de superficie aplicados después de la cocción.

\section{Obtención y preparación de la arcilla}

A pesar de existir diferentes tipos de greda en el territorio, únicamente se utilizan aquellas que se encuentra en zonas inundadas como las orillas de las lagunas o en los esteros donde aparecen balsas de agua. El lugar donde la alfarera prepara, modela y cuece la arcilla se encuentra a unos 5 kilómetros de distancia del 

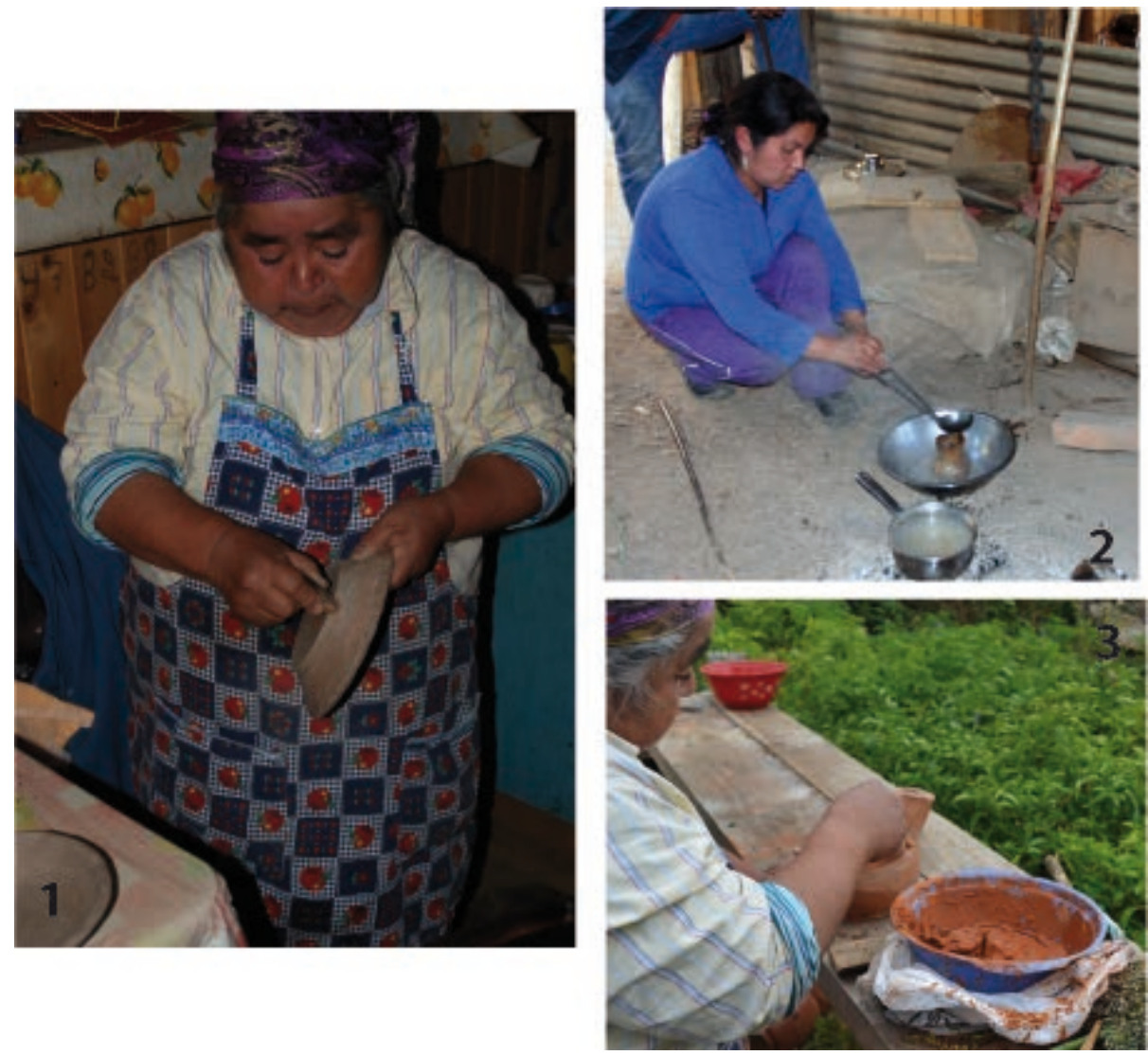

Figura 5. Tratamientos de superficie secundarios: Sopa de Lokro, bruñido y aplicación de engobe.

lugar de extracción, de la mina de arcilla. Esta se utiliza, normalmente, durante generaciones y presenta una profundidad media de entre uno y dos metros, recogiéndose. únicamente aquella greda que se encuentra en estado claramente húmedo.

Independientemente de la zona en que se encuentren, las alfareras han adoptado diferentes estrategias para tratar las arcillas: eliminación de impurezas sin adición de otros materiales, mezclas con otras arcillas, incorporación de laja molida (üku) o arena (García Rosselló y de Carvalho 2015). Incluso en algún caso se remoja la arcilla con una infusión fría de $m a q u i^{11}$ (de Carvalho y García Rosselló 2012). Todo el proceso de mezcla, amasado y preparación de la pasta se desarrolla pisando los materiales dentro de una batea de madera (o sobre un saco, en épocas más recientes). Según consta en los registros documentales, estas dinámicas técnicas se han reproducido al menos desde finales del siglo XIX (García Rosselló 2009).

\section{El modelado y los tratamientos de superficie} primarios

Nos referimos ahora a los procesos de confección de la pieza que la dotan de una forma determinada (García Rosselló y Calvo 2014; García Rosselló y Calvo 2013). La base se prepara a partir de la confección de un disco de arcilla que se inicia al aire y después se finaliza sobre un soporte de madera o, antiguamente, sobre un plato de arcilla, dejando el arranque del cuerpo ya elevado o simplemente sin iniciar. La forma del cuerpo se consigue mediante urdido de rollos aplastados, colocados en cabalgadura externa y dispuestos horizontalmente. El aplastado de los rollos es muy característico del urdido mapuche: el mismo se aplasta sobre una tabla y se estira una vez colocado sobre el cuerpo en construcción, obteniendo una forma marcadamente ovalada. Si bien la técnica es similar, es en la gestualidad de movimientos donde podemos encontrar algunas variaciones. Para la unión de los diferentes rollos al- 
gunas alfareras emplean espátulas de madera o concha, y desde 1930 se ha ido introduciendo progresivamente el uso de cucharas metálicas. Para la confección y doblado del labio se aplica un colombino en cabalgadura externa y pueden utilizarse exclusivamente las manos, alguna espátula o un trozo de cuero o de plástico. Después se pulen o compactan las paredes de la vasija, utilizando la misma herramienta que se ha empleado para unir los colombinos o las que se van a emplear para el bruñido posterior, como el canto rodado (Fig. 4).

\section{El secado}

El secado para conseguir unas paredes más consistentes y sobre las que se puedan aplicar otros tratamientos de superficie se realiza tanto dentro de la vivienda (ruka), cerca del fogón y bajo techo, como en el exterior, ya sea a la sombra o al sol. Estas actuaciones dependen de la propia alfarera y de las condiciones climáticas marcadamente cambiantes.

Tratamientos de superficie secundarios antes de la cocción

Después del secado se realiza el bruñido, una actuación común y característica de toda la cerámica mapuche. Para ello, se utilizan cantos rodados procedentes de los ríos y lagunas de la zona y se bruñe toda la superficie mediante movimientos diagonales de arriba abajo, igual que en el pulido (lustrado de la arcilla para dejar la superficie lisa y compacta).

Otra actuación que, si bien se conoce desde 1900, nunca ha tenido una aceptación homogénea entre todas las alfareras es la aplicación de un engobe por toda la superficie exterior (Fig. 5).

\section{Cocción}

Antes de cocer las cerámicas, se las va secando alrededor de estructuras de combustión, acercándolas progresivamente al fuego. El proceso se puede llevar a cabo en torno al hogar de la vivienda (ruka) o alrededor de la estructura de combustión donde se cocerán las cerámicas posteriormente. En el mundo mapuche existen dos tipos de estructuras de cocción: la cocción en fosa y la cocción de superficie. Este primer tipo de cocción ya fue referenciada en el siglo XVIII (Molina 1776, en García Rosselló y de Carvalho 2015) y es la más extendida. No obs- tante, la cocción en fosa se está abandonando progresivamente a favor de la de superficie. El combustible tradicionalmente usado ha sido la leña de hualle y las astillas de pellín, es decir maderas nativas. En la actualidad, en algunos casos se utiliza bosta de animales, pino y eucaliptos ante la invasión de tierras y destrucción del bosque nativo por parte de empresas forestales.

\section{Tratamientos de superficie secundarios poste-} riores a la cocción

Con posterioridad a la cocción algunas alfareras aplican un baño de materia orgánica en caliente, lo que provoca que el líquido llegue a temperatura de ebullición al entrar en contacto con la vasija. Existen diferentes preparados que se utilizan en el presente y se han utilizado en el pasado: leche, muday ${ }^{12}$, agua o sopa de chükül o Tükun Kachilla ${ }^{13}$ (Fig. 5).

\section{Dinámicas a escala interna: interacción entre las operaciones técnicas}

Hemos expuesto hasta aquí la existencia de una misma tradición tecnológica que presenta un alto dinamismo en términos de herramientas, materias primas y operaciones técnicas (como la aplicación de engobe o baño de materia orgánica) y en el uso de estructuras de combustión. Este marcado dinamismo se da en aquellas operaciones auxiliares (o no estructurales), que no suponen en ningún caso la trasformación de otros elementos técnicos dentro de la cadena operativa de fabricación, ni los materiales, infraestructuras o espacios en las que se realiza la actividad (ver por ejemplo García Rosselló 2008 para el norte del BíoBío). Por ello, el cambio en una de estas operaciones no supone profundas trasformaciones en la secuencia e interacción de las distintas acciones que componen la cadena operativa.

Hay que entender la variabilidad existente en estas operaciones como un alto dinamismo dentro de una misma práctica y tradición técnica, que forma parte de lo aprendido y es característico del savoir faire del grupo. La consideramos algo intrínseco y propio de la tradición cerámica mapuche, que no responde a distintos territorios (Gelbert 2003, Gosselain 2002), redes de aprendizaje (Calvo et al. 2014, Arnold 1989), grupos familiares, divisiones de género o tramas de contacto entre alfareras 
con intereses afines (Bowser 2000). Tampoco lo podemos adscribir estricto sensu a un proceso reciente de innovación, ya que la mayoría de estrategias técnicas son conocidas desde principios del siglo $\mathrm{XX}$, momento en que se inician las mayores trasformaciones en el mundo mapuche (García Rosselló 2007, 2008, García Rosselló y de Carvalho 2015). Ningún grupo de alfareras, entendido este como el perteneciente a cada una de las diferentes reducciones/comunidades, tiene conocimiento de la existencia de los otros, pero incluso una propia alfarera hace diferentes elecciones -dentro de las posibilidades existentes- durante su práctica diaria.

\section{Interacción de los procesos tecnológicos: la transversalidad tecnológica}

La tecnología cerámica mapuche se caracteriza por ser una práctica fundamentalmente manual que apenas necesita de herramientas y materias primas procesadas, convirtiéndose así, en una actividad marcadamente autónoma y oportunista donde no se requiere de herramientas o infraestructuras. La alfarera controla y conoce todo el proceso y apenas utiliza materiales y herramientas que requieran trasformaciones técnicas o ayuda externa. Algunas mujeres, además de la arcilla, tan sólo necesitan un medio de trasporte para ir a recoger la materia prima (en general se siguen utilizando bueyes y carreta, pero puede ser cualquier otro vehículo) un lugar de trabajo, que siempre es en torno a la ruka, ya sea dentro o fuera. En este último caso, es de especial importancia el fogón que configura el interior de la vivienda mapuche y el combustible para la cocción, que se obtiene de los bosques alrededor de la vivienda. Las alfareras que no utilizan solo las manos para modelar, simplemente incorporan espátulas de madera que adaptan ellas mismas. A su vez, todas incorporan productos que conocen muy bien y que forman parte de su vida cotidiana y su aprendizaje, como los frutos de la huerta (por ejemplo, hojas de maqui), o platos alimenticios para tratar las superficies en el caso de la sopa de locro y el muday ${ }^{14}$.

Si bien la práctica alfarera no interactúa estrechamente con otras tecnologías, existen otras esferas técnicas que no escapan de la misma lógica técnica y social. Se trata de prácticas que participan de una tradición tecnológica común a todas las esferas sociales que se man- tiene, con algunas transformaciones a lo largo del tiempo desde la llegada de los españoles. Son prácticas en las que también es inherente un alto grado de dinamismo en algunas actuaciones técnicas de tipo no estructural. Y en la que si bien se han modificado parte de los productos finales, resultado de la acción sobre la materia, también han perdurado otros, y se han mantenido las mismas técnicas de fabricación. Todo ello, dentro de un contexto de aprendizaje no restringido en el que no participa toda la población, trasmitido dentro de redes de comunicación familiar y doméstica, y asociado a la división de género.

El telar mapuche, por ejemplo, ha mantenido tanto la estructura (telar de marco o witral) como las técnicas tradicionales, a la vez que ha ido incorporando nuevas formas y motivos fruto de nuevas demandas. Mantiene como materia prima la lana y existen numerosas estrategias técnicas de urdimbre que no son utilizadas por todas las tejedoras. Igual que la alfarería, es una actividad exclusivamente femenina que se desarrolla dentro de la casa, alrededor del fogón, con una baja visibilidad social y que se alterna con otras actividades domésticas. Se requiere únicamente de una infraestructura básicamente manual donde apenas se utilizan herramientas, junto con otra procedente del entorno cercano, como las maderas para el witral, la lana y los pigmentos naturales para el teñido (Joseph 1931; Mege 1990; Wilson 1993). No obstante, no requiere de la interacción con otras tecnologías, pues la tejedora puede confeccionar la infraestructura necesaria con los productos que encuentra en su entorno cercano. Al igual que la cerámica, solo el resultado final es visible por el resto del grupo. Por el contrario, las formas y diseños (es decir, el producto obtenido) están profundamente conectados con las técnicas utilizadas. Así, según la técnica de urdimbre utilizada se obtiene uno u otro motivo decorativo, lo que determinará el tipo de prenda confeccionada.

Otro caso similar es el de la construcción de la vivienda $(r u k a)$. Al contrario de las actividades anteriormente descritas, esta práctica es esencialmente masculina y comunitaria. En su construcción participa parte de la comunidad bajo las órdenes de un conocedor de la técnica, que la ha aprendido dentro de las redes de trasmisión de conocimiento familiares. Pese a las diferencias existentes con las producciones realizadas por mujeres al ser una actividad comunitaria con una alta visibilidad social ajena 

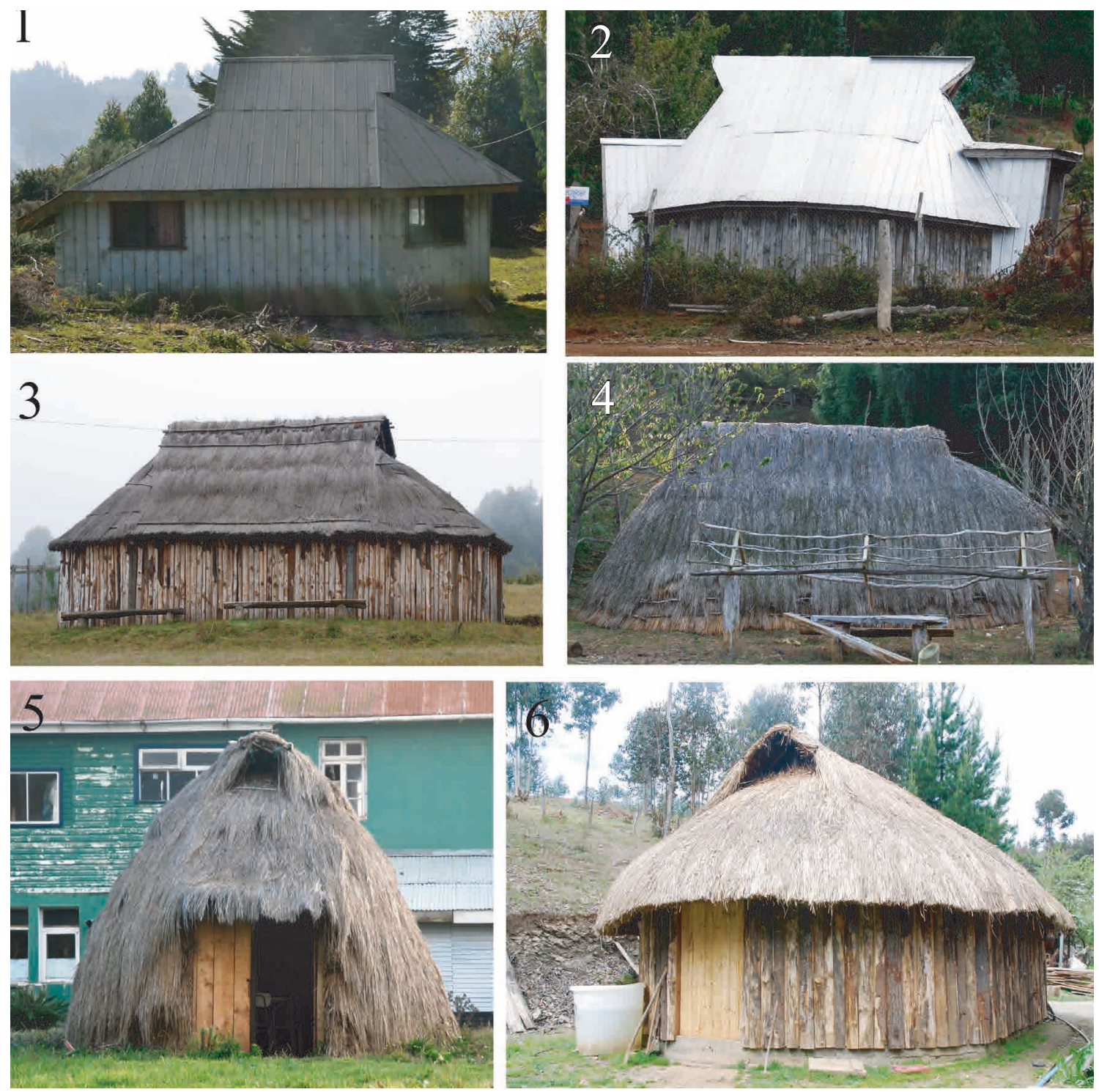

Figura 6. Tipos de vivienda mapuche inspirados en la ruka tradicional: 1.- Lago Budi (Puerto Saavedra).

2.-Lago Lleu-Lleu (Contulmó). 3.- Elicura (Contulmó). 4.- Lago Budi (Puerto Saavedra). 5.- Puerto Saavedra. 6.- Dibulko 2 (Lumaco).

a la práctica diaria, existen algunas similitudes con dichas actividades que se pueden relacionar con la lógica social y técnica existente en el pueblo mapuche. Se trata igualmente de una actividad doméstica, donde participan diferentes miembros de la comunidad vinculados por lazos de parentesco y donde el conocimiento no está generalizado, por lo que la mayoría de miembros están bajo las órdenes de un miembro destacado de la comunidad. Constituye asimismo un saber que ha perdurado en el tiempo, si bien se han ido incorporando nuevas técnicas y materiales. Existen diferentes formas y tamaños de vivienda: desde aquellas que presentan una base circular a otras poligonales o en forma de U. Algunas se fabrican con maderas nativas y arbustos del entorno, como el copihue, el pellín, el canelo o el boqui para amarrar, y paja o totora para cubrir el techo. Otras utilizan tablas para los costados, e incluso algunas tienen cubierta de zinc (Fig. 6). No obstante, todas ellas siguen la misma estructura habitacional alrededor del fogón, con un armazón interior visto sobre el que se coloca la techumbre, con una forma de tendencia ovalada, el techo inclinado y dimensiones adaptadas al tamaño de la familia. Además, no presentan ventanas pero tienen una entrada principal 
orientada al este y aberturas laterales en el techo de tipo triangular que permiten la salida del humo. En la actualidad, la mayoría de las ruka tienen la función de cocina, sala de estar y lugar donde se desarrollan las actividades artesanales, mientras que los dormitorios se encuentran en una estructura contigua (Joseph 1928; Sepúlveda 2013).

\section{Interacción entre dinámicas técnicas y ac- ciones sociales: flexibilidad social y organi- zación territorial}

Como hemos comentado anteriormente, creemos que existe una correlación entre muchas de las prácticas técnicas mapuche y las dinámicas sociales. De hecho, no es que las prácticas técnicas sean una expresión de las dinámicas sociales, sino que participan de ellas al mismo nivel, las estructuran y a la vez son estructuradas por ellas dentro de la práctica diaria.

En este sentido, vamos a profundizar en aquellas dinámicas sociales que se vinculan con un alto dinamismo técnico dentro de la homogeneidad. Una homogeneidad que está presente en la sociedad mapuche desde antes de su sometimiento y concentración en reservas por parte del Estado Chileno en el siglo XIX (Bengoa 1991, 2003), llegando a constituirse en tradición nucleada a través de la organización entorno a la ruka (Adán 2014). La estructura social y la organización territorial son precisamente dos de las esferas que más participan de este alto dinamismo y flexibilidad característica de la sociedad mapuche.

Una sociedad que ha sufrido toda una serie de cambios en los últimos siglos que significaron la trasformación y, sobre todo, la adaptación y resiliencia de muchas de las manifestaciones, estructuras y modos de pensar que caracterizaban a los grupos presentes en el Gulumapu antes del contacto con las huestes castellanas (Boccara 1999, Sauer 2015). Durante más de trescientos años, algunos grupos indígenas situados al sur del Bío-Bío se vieron obligados a adoptar estructuras, materiales y prácticas sociales de tipo militar destinadas a contener el avance de los colonizadores (Jara 1984, Foerster 1995, Boccara 1999, Paño Yánez 2005, Marimán et al. 2006, Le Bonniec 2009). Por ejemplo, la adopción del caballo; la agrupación en ayllarehue (nueve rehue) y butalmapus (diferentes ayllarehue) dirigidas por un toqui, que era un jefe militar pero no tenía más poder que el otorgado por los diferentes linajes que conformaban las agrupaciones; o la reunión en parlamentos para establecer políticas de alianzas con los poderes coloniales.

Esta situación se mantuvo hasta la mal llamada "pacificación de la Araucania" (18611883), cuando el Estado Chileno conquistó y expropió el territorio nativo, recluyendo a las familias mapuche en reducciones (Course 2011). A partir de 1883 el Estado Chileno concentró a los diferentes grupos en reducciones, otorgando títulos de merced al jefe o representante del grupo. A partir de 1979 la reducción deja de ser una figura legal entregándose títulos de propiedad individual sobre la tierra. Ello supuso la parcelación de las reducciones en terrenos de una extensión media que no superaba las 20 hectáreas (Stuchlick 1974).

Las reducciones supusieron la eliminación de un patrón de residencia disperso y la movilidad de las familias, al estar controladas por el Estado (Paño Yañez 2005). Además, implicó la incorporación de la ganadería y su dependencia económica que se combinaba con la agricultura, la caza y la recolección (Bengoa 2003). No obstante, muchas de las organizaciones sociales de las que tenemos plena constancia a partir de los documentos del siglo XVIII (Zavala 2011, Adán 2014) y especialmente en el XIX (Bengoa 2003) no han desaparecido, sino que han trasformado su función política y militar (Montecino 1980, Bengoa 1991, Foerster 1995, Campbell 2012, Adán 2014) para convertirse en manifestaciones vinculadas a rogativas relacionadas con los distintos linajes y el culto a los ancestros (Fernández 1982, Foerster 1995, Course 2011, Dillehay 2011). Incluso las reglas y normas sociales (admapu) que se establecieron en el pasado siguen teniendo hoy vigencia, aunque sea de tipo moral, religioso e identitario más que jurídico (Stuchlick 1974; Boccara 1999). De hecho, la organización social en comunidades pequeñas, dispersas y autónomas no sometidas a un poder centralizado, características de tiempos anteriores al siglo XIX y organizadas a partir de la ruka se ha mantenido dentro del confinamiento de la reducción (Faron 1969, Course 2011, Adán 2014).

\subsection{Organización territorial y social}

La conceptualización del espacio establecida por el mapuche es significativamente diferente a la de tradición europea. En ella interac- 
túan el espacio geográfico tangible (puntos cardinales), el social, que sitúa a la familia en el centro de cualquier interacción con los demás, y el espacio imaginado, no tangible y de tipo simbólico que incluye tanto a los espacios rituales como al mundo sobrenatural. No obstante, siempre está en el centro la familia y la mapu-tierra (Course 2011, Dillehay 2011, Adan 2014).

La propia cosmovisión del mapuche no está vinculada a un espacio completamente territorializado, es decir, no está directamente vinculado con la propiedad de un lugar, con la asociación de los ancestros del linaje a un lugar específico o con patrones de residencia estables (Adan 2014). Dicha conceptualización del espacio sitúa a la familia y al linaje masculino en una posición central (Faron 1969, Course 2011).

Incluso con la concentración en reducciones, los mapuche nunca han constituido su residencia en aldeas o pueblos, prefiriendo el asentamiento disperso unifamiliar en un conjunto de viviendas más o menos separadas respecto a otros miembros del grupo. A lo largo de los últimos siglos ello ha contribuido también a mantener su autonomía e independencia, y ciertas costumbres propias (Dillehay 1990, Boccara 1999, Bengoa 2003, Le Bonniec 2009, Adan 2014).

La familia extensa juega un papel central tanto en la manera de entender el mundo como en las relaciones sociales. La organización social ha carecido, en general, de unidades mayores de parentesco que los grupos locales. Unos grupos cohesionados en torno a reglas de descendencia patrilineal y de residencia patrilocal (Faron 1969, Course 2011, Campbell 2012, Adán 2014), que suponen que la mujer abandona su territorio y unidad familiar para pasar a residir con la familia del esposo, compuesta por sus padres, hijos varones con sus esposas e hijos, y hermanos y hermanas solteros. Todos ellos pueden vivir juntos o en diferentes rukas vecinas. Esta organización resulta fundamental para entender la autonomía política, económica y social de estos grupos y de sus expresiones materiales, tal como ocurre con las prácticas técnicas.

A lo largo de su historia, ni en momentos prehispánicos, ni durante los enfrentamientos con las tropas castellanas, ni durante la conquista territorial chilena, los grupos mapuche han adoptado un tipo de organización social y económica sometida a un poder centralizado; más bien, al contrario, han mantenido su celosa independencia dentro del linaje, conservando patrones de residencia dispersos y una economía autosuficiente.

Tanto en el pasado como en la actualidad, un grupo de familias relacionadas en torno a un antepasado común se integra en una unidad social más amplia, como el lof. Antes de la concentración en reducciones, esta unidad no tenía necesariamente que obedecer a criterios territoriales, si bien en muchas ocasiones estaba constituido por las casas vecinas que ocupaban un pequeño territorio. Era una organización de ayuda mutua a la cabeza de la cual estaba un longko que representaba a un mismo linaje común. Un ejemplo más de la diversidad existente en el mundo mapuche.

En la actualidad, la figura del lof sigue funcionando ya que la mayoría de los confinamientos reduccionales se organizaron a partir de vínculos y relaciones familiares (Stuchlick 1974).

Existían agrupaciones sociales mayores que se conformaban para afrontar calamidades climáticas o conflictos armados (rehues, aillahures y butalmapus), muchas de las cuales se siguen utilizando para el desarrollo de ceremonias rituales en torno a un linaje común (Faron 1969; Grebe 2006, Dillehay 2011, Adán 2014). Estas agrupaciones sociales se disolvían de común acuerdo ya que su estructuración no respondía a normas establecidas y rígidas. De esta forma, estaban en constante construcción y trasformación (Zavala 2011).

En el mundo mapuche no existen relaciones sociales estables y reguladas fuera de la familia, lo que supone una cierta autonomía, más allá de los contactos esporádicos dentro del territorio cercano, fruto de actividades de subsistencia y el desarrollo de manifestaciones comunitarias de carácter ritual (Course 2011). La concentración de las distintas familias y linajes en reducciones a partir de la ocupación chilena de las tierras (1880-1910), ha acrecentado mucho más su aislamiento (Stuchlick 1974; Bengoa 1991, 2003).

En este sentido, muchos mapuche que no interactúan con otros territorios y grupos no consiguen establecer una conceptualización global de su cultura, lo que junto a su marcado regionalismo y sedentarismo actual, facilita la existencia de versiones parciales de conocimientos y experiencias que tienden a reflejarse en todas sus prácticas (Grebe 2006, Course 2011). Este marcado aislamiento, los contac- 
tos sociales esporádicos y la baja visibilidad social de muchas de las prácticas técnicas que desarrollan los mapuche condicionan una alta variabilidad que hemos comentado y las constantes adaptaciones de dichas prácticas. Hablamos de una sociedad que vivía y vive en parcialidades autónomas, generalmente unidas por relaciones de parentesco en base a un linaje común, pero que se agrupa de forma temporal y coyuntural para la realización diversas labores productivas e/o ideológicas (Boccara 1999, Faron 1969, Adan 2014, Bengoa 1991, 2003). Todo ello supone el mantenimiento del derecho de cada familia a decidir de forma autónoma e independiente.

\subsection{Autonomía y aprendizaje}

Proponemos que el dinamismo que estamos enfatizando aquí está enraizado en la propia manera de ver el mundo de los grupos mapuche y es una forma intrínseca de sus manifestaciones sociales y culturales a través de una característica autonomía (Bengoa 1991, 2003, Campbell 2012, Adán 2014) y ligazón con su linaje (Faron 1969, Course 2011). El carácter libre e independiente del pueblo mapuche está propiciado por su propia identidad, manera de entender el mundo, de organizarse en sociedad y de participar de las manifestaciones culturales y religiosas. Todo ello se basa en una forma propia de trasmitir los conocimientos y adoptar las tradiciones a nuevas realidades. En este sentido, no podemos dejar de lado, como elemento fundamental, el modo en que se trasmiten a través del tiempo y se generalizan por el territorio los conocimientos e ideas que con el paso de los años se convierten en tradición. Hay por ello, dos fenómenos que se vinculan muy bien con estas reflexiones que estamos defendiendo sobre la tradición y la autonomía:

1.- La ausencia de una autoridad de carácter religioso, social o político centralizada, que se responsabilice de velar por el control de las ideas y la trasmisión de conocimientos. Incluso en la religión y cosmovisión mapuche se observa la ausencia de un poder claramente definido, tanto en el mundo celestial como en el terrenal (Boccara 1999; Grebe 2006, Course 2011). El longko mapuche, representante de un linaje, por ejemplo, constituye un tipo de jefatura muy particular. En este caso, la fuente de su poder recae en el servicio a los suyos, su prestigio en la resolución de conflictos, su capacidad para aconsejar, su sabiduría y, sobre todo, la manera en que se comporta y expresa como moderador durante las discusiones que preside. En ningún caso esta figura ejerce control y poder sobre los miembros del grupo (Marimán et al. 2006). Por ello, la autoridad sobre su linaje es significativamente débil en términos de poder, control y coerción. De hecho no hay longko, u otro tipo de figura representativa, fuera de la unidad familiar, que controle o tenga influencia en otros territorios o grupos. Todo ello condiciona que la trasmisión de conocimientos e ideas no circule más allá de los lof $\mathrm{y}$, excepcionalmente, en los rewes o ayllarehue con los que interacciona la familia y el linaje.

2.- La manera en que los grupos mapuche han educado a sus hijos e hijas y han trasmitido sus conocimientos, generación tras generación, tiene también mucho que ver con el dinamismo y la diversidad social y tecnológica existentes.

Antes de la escolarización generalizada de niños y niñas indígenas por parte del Estado Chileno, eran determinadas personas, conocidas como kimche $e^{15}$, las que instruían a los hijos de la familia por medio de un discurso informal (Quilaqueo 2012), en el cual incorporaban al conocimiento colectivo algunos saberes propios. En estos discursos, generados dentro de las actividades diarias de la familia, los diferentes participantes podían intervenir, sociabilizándose y construyendo su propio discurso sobre una base común (Quilaqueo 2005, 2012).

De hecho, se ha planteado que el aprendizaje mapuche es holístico, vivencial y claramente subjetivo (Quilaqueo 2005), que busca formar una identidad autónoma y propia. De esta forma, los niños asimilaban los diferentes aprendizajes y conocimientos desde la más tierna infancia mediante la participación en las tareas que llevaban a cabo los adultos. Así experimentaban las distintas actividades e imitaban a sus familiares cercanos. Durante este proceso no eran corregidos por los adultos con el objetivo de que cada uno encontrara su propio camino (Mineduc 2002).

\section{Conclusiones}

La idea de que detrás de una lógica técnica hay una lógica social no es algo nuevo. Tampoco hay nada nuevo en una propuesta que incide en la interrelación existente entre técnicas, tecno- 
logías y grupos sociales. Sin embargo, nos parece que puede resultar interesante para futuras discusiones interpretativas sobre la sociabilización de las técnicas, mostrar cómo esta perspectiva ha resultado sumamente útil para visualizar la manera en que, en el caso mapuche, el dinamismo de las técnicas cerámicas se puede correlacionar con un fenómeno mucho más amplio que incluye otras tecnologías y las propias dinámicas sociales y territoriales del grupo.

Esto es posible gracias a un proceso de aprendizaje en el que los niños y niñas están presentes desde muy jóvenes en las actividades familiares, incorporando progresivamente y de forma inconsciente esquemas de percepción, estrategias de actuación o convencionalismos sociales propios del grupo. En definitiva, mediante la naturalización social, vehiculada a través de la práctica diaria, se conforman los esquemas mentales del grupo que, para el caso que nos ocupa, suponen, entre otras cosas, una alta capacidad de adaptación, negociación y flexibilización ante las diferentes situaciones sociales.

Paralelamente, desde la década de 1990 se está desarrollando un fenómeno de renacimiento cultural y reivindicación política (Boccara 2006) donde aquellos que supuestamente detentan la hegemonía cultural (sensu Gramsci 2013) como los intelectuales, líderes indígenas y representantes sociales, construyen discursos que representan un mundo mapuche armónico, inalterado y uniforme, mientras que la mayoría de los subalternos de este mismo grupo no comparten dicho capital cultural y utilizan categorías locales que no suponen la adopción de una misma cosmovisión, historia o territorialidad (Le Bonniec 2009), e incluso difieren de esta construcción imaginada del pasado $\mathrm{y}$ el territorio.

Todo ello no es más que otro ejemplo del alto dinamismo social y local entre los mapuche, una situación en la que las elites no tienen el control o la representación directa de las poblaciones, más allá de la supuesta representación y hegemonía cultural.

Por ello, hemos puesto el énfasis en la diversidad existente en el mundo mapuche, visibilizada a través de su materialidad y práctica técnica, intentando huir de modelos y planteamientos simplistas y uniformizadores. Y, ante todo, destacando que dentro de la evidente homogeneidad cultural mapuche existe gran diversidad en multitud de esferas.

En este sentido, la materialidad, y las prácticas técnicas tienen mucho que decir en un mundo en continua transformación y desestructuración, donde las artesanías se mantienen como medio de vida entre los grupos subalternos y como símbolo de un pasado imaginado y fosilizado entre las elites culturales que influyen en las políticas públicas.

\section{Agradecimientos}

A todos los informantes que han colaborado en este trabajo. Especialmente a las alfareras mapuche por su disponibilidad, generosidad y hospitalidad: Rosa y Selimina Huaquil, Ema y Maria Tranamil, Sandra Rain (Dibulko 1 y 2, Lumaco); María Pucol y Lucinda Antil (Elicura, Contulmó); Fresia Nahuelque, Agustina Calvellunca, Luisa Manilio (Huallepen Bajo); Maria y Juana Collenco (Aniñir, Ercilla); María Cachaña, Juana Luisa Machacón, Gladys Comillo (Pilmaiquenco, Carahue); Ana María y Rosa Aguayo, Rosa Gallardo, Dominga Neculmán (Roble Huacho); Margarita Quilaqueo (Balsa Pocayan, Teodoro Schmith); Helvetia Cayupan (Pillanlebún, Lautaro). A Manuel Calvo, Aixa Vidal, Javiera Letelier y Carolina Caballero por su colaboración científica y personal. Los datos e imágenes presentados en él cuentan con la conformidad de personas y autores.

\section{Notas}

2. Noviembre 2007 en las comunas de Valdivia, Freire, Padre las Casa y Temuco (junto a C. Caballero); octubre 2011 en las comunas de Lumaco, Los Sauces, Purén, Cañete y Contulmo (junto G. de Carvalho); abril 2013 en las comunas de Puerto Saavedra, Padre las Casas, Carahue, Tiruá, Chol Chol y Contulmó (junto a M. Calvo); enero y febrero 2014 en las comunas de Pucón, Villarrica, Cunco, Temuco, Cañete, Angol, Purén, Lumaco, Traiguen, Ercilla, Galvarino, Curarrehue, Collipulli, Mulchen, Renaico, Loncoche y Gorbea (junto a C. Caballero); mayo-septiembre 2015 en las comunas de Cunco, Cholchol, Pitrufquen, Freire, Temuco, Padre las Casas, Pangupulli (con la coordinación de J. Letelier y la participación de V. Vega y un grupo de alumnos de la carrera de arqueología de la Universidad Alberto Hurtado); noviembre 2015 
en las comunas de Puerto Saavedra, Teodoro Schmith, Padre las Casas, Vilcun, Melipeuco, Nueva Imperial, Carahue (junto a J. Letelier y V. Vega).

23 Rosa y Selimina Huaquil, Ema y Maria Tranamil, Sandra Rain (Dibulko 1 y 2, Lumaco); María Pucol y Lucinda Antil (Elicura, Contulmó); Fresia Nahuelque, Agustina Calvellunca, Luisa Manilio (Huallepen Bajo); Maria y Juana Collenco (Aniñir, Ercilla); María Cachaña, Juana Luisa Machacón, Gladys Comillo (Pilmaiquenco, Carahue); Ana María y Rosa Aguayo, Rosa Gallardo, Dominga Neculmán (Roble Huacho); Margarita Quilaqueo (Balsa Pocayan, Teodoro Schmith); Helvetia Cayupan (Pillanlebún, Lautaro).

4. Cissus striat. Planta trepadora que se utiliza para cestería en el centro-sur de Chile.

5. La "reducción" es el lugar donde el estado chileno, después de conquistar y ocupar el territorio en el siglo XIX y XX, confinó a las familias mapuches, otorgándoles pequeñas parcelas en zonas improductivas (principalmente en marismas y zonas montañosas escarpadas), mientras que las mayores extensiones de tierras -las más productivas- fueron cedidas a inmigrantes extranjeros (principalmente alemanes, suizos e italianos).

6. Año nuevo mapuche.

7. Ritual de curación protagonizado por la curandera o machi.

8. Rogativa comunitaria dirigida por la machi.

9. Winka: En mapundungun: "extraño", "invasor". Por extensión, " no mapuche”.

10. Vasija hemisférica y de forma abierta usada para preparar pastel de choclo (maíz) y de usos extendido por todo Chile (por ejemplo García Rosselló 2006 y 2008).

11. Aristotelia Chilensis. Arbol nativo que se utiliza por sus propiedades medicinales.

12. Chicha mapuche. Bebida alcohólica fermentada a base de cereales, mote o patata.

13. Trigo tostado en una callana y ligeramente molido. Locro en chileno.

14. Chicha mapuche. Bebida alcohólica fermentada a base de cereales, mote o patata.

15. Los kimche siguen teniendo un papel fundamental en la trasmisión de conocimientos pero en la actualidad esta se complementa e incluso compite con la educación escolar obligatoria impuesta por el Estado Chileno. Si bien la ley de educación primaria de 1920 incluía a los mapuches, en las parcialidades donde hemos trabajado esta no fue efectiva hasta bien entrado en siglo XX.

\section{Bibliografía}

Adán, L. (2014): Los reche-mapuche a través de su sistema de asentamiento (S. XV-XVII). Tesis doctoral para optar al grado de Doctora en Historia. Mención Etnohistoria. Facultad de Filosofía y Humanidades. Santiago de Chile.

Adán, L.; Mera, R.; Munita, D.; Alvarado, M. (2016): Análisis de la cerámica de tradición indígena en la jurisdicción de Valdivia: estilos Valdivia, Tringlo y decorados con incrustaciones. Arqueología de la Patagonia: de mar a mar. (Mera, F., ed.) Ediciones CIEP y Nire Negro Ediciones, Santiago de Chile: 313-323.

Alvarado, M. (1997): La tradición de los grandes cántaros: reflexiones para una estética del envase. Revista Aisthesis, 30: 105-124.

Arnold, D. (1989): Patterns of learning residence and descent among potters in Ticul, Yucatán, México. Approaches to cultural identity (S. Shennan, ed.) Unwin Hyman, Londres: 174-184.

Bengoa, J. (1991): Historia del pueblo mapuche. Colección de Estudios Históricos, Ediciones Sur, Santiago de Chile.

Bengoa, J. (2003): Historia de los antiguos mapuches del Sur. Desde antes de la llegada de los españoles hasta las paces de Quilin. Catalonia, Santiago de Chile.

Boccara, G. (1999): Etnogénesis mapuche: resistencia y reestructuración entre los indígenas del centro sur de Chile (siglos XVI-XVIII). Hispanic American Historical Review, 79-3: 425-461.

Boccara, G. (2006): The brighter side of the indigenous renaissance. Nuevo Mundo Mundos nuevos, 6. (Débats). [URL: http://nuevomundo.revues.org/2405] [Actualizada el 16/6/2006]. Acceso el 1/4/2016. http://dx.doi.org/10.4000/nuevomundo.2405

Bowser, B.J. (2000): From pottery to politics: An Ethnoarchaeological study of political factionalism, ethnicity, and domestic pottery style in the Ecuadorian Amazon. Journal of Archaeological Method and Theory, 7-3: 219-248.

Calvo, M. y García Rosselló, J. (2014): Acción técnica, interacción social y práctica cotidiana: Propuesta interpretativa de la tecnología en la prehistoria. Trabajos de Prehistoria, 71-1: 7-22. 
Calvo, M., García Rosselló, J., Javaloyas, D., y Albero, D. (2014): Playing with mud? An ethnoarchaeological approach to children's learning in Kusasi ceramic production. Children, identity and space. (M. Sanchez Romero, E. Alarcón, G. Aranda, eds.), SSCIP Monograph Series, Oxbow, Oxford: 88-104.

Campbell, R. (2012): Cambio cultural, unidad doméstica y comunidad: la Araucanía a la luz del registro etnohistórico (y arqueológico). Actas del XVIII Congreso Nacional de Arqueología Chilena, Sociedad Chilena de Arqueología: 359-368.

Carvalho-Amaro, G. de y García Rosselló, J. (2012): Cadena operativa y tecnología cerámica. Una visión etnoarqueológica de las alfareras mapuche de Lumaco. Boletín de la sociedad Chilena de Arqueología, 41-42: 53-79.

Course, M. (2011): Becoming Mapuche. Person and Ritual in Indigenous Chile. University of Illinois Press, Champaign.

Dillehay, T. (1990): Araucania. Presente y Pasado. Andrés Bello, Santiago de Chile.

Dillehay, T. (2011): Monumentos, imperios y resistencia en Los Andes. El sistema de gobierno Mapuche y las narrativas rituales. Universidad Católica del Norte y Universidad de Vanderbilt, Ocho Libros Editores, Santiago de Chile.

Faron, L. (1969): Los mapuches. Su estructura social. Instituto Indigenista Americano, México.

Fernández, A. (1982): Rogativas Mapuches. Amerindia, 7: 109-144.

Foerster, R. (1995): Introducción a la religiosidad mapuche. Editorial Universitaria, Santiago de Chile.

Gándara, M. (2006): La Inferencia por analogía: más allá de la analogía etnográfica, Treballs d'Etnoarqueologia, 6: 13-24.

García Rosselló, J. (2006): La producción cerámica en los valles centrales de Chile: estrategias productivas. Treballs d'Etnoarqueologia, 6: 297-313.

García Rosselló, J. (2007): La producción cerámica mapuche. Perspectiva histórica, arqueológica y etnográfica. VI Congreso Nacional de Antropología, Colegio de Antropólogos, Valdivia.

García Rosselló, J. (2008): Etnoarqueología de la producción cerámica. Identidad y territorio en los Valles Centrales de Chile. Mayurqa, 32. Número monográfico.

García Rosselló, J. (2009): Tradición tecnológica y variaciones técnicas en la producción cerámica mapuche. Complutum, 20-1: 153-171.

García Rosselló, J. (2011): Modelado, aprendizaje y espacio social: una reflexión desde la tecnología cerámica. Werken, 14: 63-74.

García Rosselló, J., Calvo, M. (2013): Making pots: El modelado de la cerámica y su potencial interpretativo. BAR International Series 2540, Archaeopress, Oxford.

García Rosselló, J.; Carvalho, A. de (2015): La cerámica mapuche a mano. Una aproximación etnográfica basada en las técnicas. Trabalhos de Antropologia e Etnologia, 53-55: 195-216.

Gelbert, A. (2003): Traditions cerámiques et emprentes techniques. Etude_Etnoarcheologique dans le haute et moyenne vallées du fleuve Sénegal. MSH, Epistèmes, París.

González-Ruibal, A. (2016): Ethnoarchaeology or simply archaeology? World Archaeology, 48 (5): $687-$ 692. http://dx.doi.org/10.1080/00438243.2016.1209125

Gosselain, O. (2002): Poteries du Cameroun meridional. Styles, techniques et rapports a l'identité. CNRS, París.

Gramsci, A. (2013): Antología. Selección, traducción y notas de Manuel Sacristán. Akal, Madrid.

Grebe, M. (1972): Cosmovisión del mundo mapuche. Cuadernos de la realidad nacional 14: 46-73.

Grebe, M. (2006): Culturas indígenas de Chile. Un estudio preliminar. Pehuén ediciones, Santiago de Chile.

Hernández, A., Ramos, N., Cárcamo, C. (1997): Mapuche. Lengua y cultura. Pehuén ediciones, Santiago de Chile.

Hernando, A., González-Ruibal, A. (2011): Fractalidad, materialidad y cultura. Un estudio etnoarqueológico de los Awá-Guajá (Maranhão, Brasil). Revista Chilena de Antropología, 24: 9-61.

Hodder, I. (1982): Symbols in action. Cambridge University Press, Cambridge.

Jara, A. (1984): Guerra y sociedad en Chile. Editorial Universitaria, Santiago de Chile.

Joseph, C. (1928): La vivienda araucana. Revista Universitaria, Año XIII. No 10.

Joseph, C. (1931): Los tejidos araucanos. Editorial San Francisco. Padre Las Casas, Chile.

Le Bonniec, F. (2009): Reconstrucción de la territorialidad mapuche en el Chile contemporáneo. Territorio y territorialidad en contexto post-colonial Estado de Chile- Nación Mapuche (J. Cabulcura, F. Le Bonniec, Eds.), Working papers series no 30. Nuke Mapuförlaget, Temuco: 44-79.

Lemonnier, P. (1992): Elements for an anthropology of technology. University of Michigan, Museum of Anthropology, Michigan.

Lemonnier, P. (1993): Technological choice. Transformations in material cultures since the Neolithic. Routledge, Londres: 1-35.

León, L. (2008): El ocaso de los lonkos y el caos social en el Gullumapu (Araucanía) 1880-1925. Cuadernos interculturales, 6-11: 172-208. 
Leroi-Gourhan, A. (1964): La geste et la Parole. Technique et Langage. Editorial A. Michel. París.

Mahias, M. (1993): Pottery techniques in India. Technical variants and social choice. Technological choices. Transformation in material culture since the Neolithic (P. Lemonnier, ed.). Routledge, Londres: 157180.

Marimán, P., Caniuqueo, S., Millalén, J. Levil, R. (2006): ¡Escucha Winka! Cuatro ensayos de historia nacional mapuche y un epílogo sobre el futuro. Lom ediciones, Santiago de Chile.

Mege, P. (1990): Arte textil mapuche. Museo de Arte Precolombino. Santiago de Chile.

Montecino, S. (1980): La sociedad mapuche entre los siglos XVI y XIX: su transformación estructural. Tesis para optar al grado de Licenciada en Antropología, Departamento de Antropología, Universidad de Chile, Santiago de Chile.

Olsen, B. (2010): In Defense of Things: Archaeology and the Ontology of Objects. Altamira Press, Lanham.

Paño Yáñez, P. (2005): El proceso histórico de las transformaciones socioculturales mapuches desde la conquista hasta el siglo XX. Boletín Americanista, 55: 206-240.

Quilaqueo, D. (2005): Educación intercultural desde la Teoría del Control Cultural en contexto de diversidad sociocultural mapuche. Cuadernos interculturales, 3-4: 37-50.

Quilaqueo, D. (2012): Saberes educativos mapuche: racionalidad apoyada en la memoria social de los kimches. Atenea, 505: 19-112.

Sepúlveda, O. (2013): Cultura y hábitat residencial: el caso mapuche en Chile. Tesis Doctoral. Universidad Politécnica de Madrid, Madrid.

Sauer, J. (2015): The Archaeology and Ethnohistory of Araucanian Resilience. Contributions To Global Historical Archaeology, Springer, New York.

Soto, E. (2009): La escolarización de los mapuche de Chile y la nueva mirada del estado. XXVII Congreso de la Asociación Latinoamericana de Sociología. VIII Jornadas de Sociología de la Universidad de Buenos Aires. Asociación Latinoamericana de Sociología: 1-10.

Stuchlick, M. (1974): Rasgos de la sociedad mapuche contemporánea. Ediciones Nueva Universidad. Universidad Católica de Chile, Santiago de Chile.

Thomas, J. (2004): Archaeology and Modernity. Routledge, Nueva York

Willson, A. (1993): Textilería mapuche. Memoria y cultura: femenino y masculino en los oficios artesanales. Cedem, Santiago de Chile.

Zavala, J. M. (2011): Los mapuches del siglo XVIII. Ciudad, Ediciones de la Universidad Católica de Temuco, Temuco. 